\title{
Ultrafast Spectroscopy of Oxyhemoglobin during Photodissociation
}

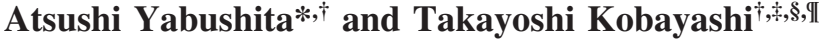 \\ Department of Electrophysics, National Chiao-Tung University, Hsinchu 300, Taiwan; ICORP, Japan Science \\ and Technology Agency, 4-1-8, Honcho, Kawaguchi, Saitama 332-0012, Japan; Department of Applied Physics \\ and Chemistry and Institute for Laser Science, University of Electro-Communications, 1-5-1, Chofugaoka, \\ Chofu, Tokyo 182-8585, Japan; and Institute of Laser Engineering, Osaka University, 2-6 Yamada-oka, Suita, \\ Osaka 565-0971, Japan
}

Received: April 21, 2010; Revised Manuscript Received: August 2, 2010

\begin{abstract}
Ultrafast time-resolved pump-probe spectroscopy was studied to clarify the detailed mechanism in the photodissociation process of oxyhemoglobin in the visible spectral range. The photodissociation had not been time-resolved and only the upper limit of the time needed for the dissociation process was claimed to be faster than $50 \mathrm{fs}$; it was time-resolved to be $45 \pm 5 \mathrm{fs}$ with $10 \mathrm{fs}$ time resolution. The broadband spectrum of the visible laser pulses enabled us to observe the signal over a broad spectral range. A broadband multichannel detector array was used to simultaneously obtain the pump-probe signal at all probe frequencies. Thus, we obtained for the first time the spectra of the ultrashort lifetime electronic excited state of $\mathrm{HbO}_{2}$ in the visible range from $523 \mathrm{~nm}\left(19109 \mathrm{~cm}^{-1}\right)$ to $719 \mathrm{~nm}\left(13914 \mathrm{~cm}^{-1}\right)$. After the photodissociation, subpicosecond time constant was found and attributed to the dynamics of the photolyzed Hb. Time-resolved difference absorption spectra in $0-100 \mathrm{fs}$ showed oscillatory motion reflecting wavepacket motion in the potential energy surface of the photoexcited $\mathrm{HbO}_{2}$ during the ultrafast photolysis.
\end{abstract}

\section{Introduction}

The rapidly growing body of data on heme proteins in the ultrafast time domain is revealing their complicated photophysics, which is sensitive to both the protein structure ${ }^{1-5}$ and to the ligand $\left(\mathrm{CO}, \mathrm{O}_{2}\right.$, and $\left.\mathrm{NO}\right){ }^{1,3-9}$ Experimental studies clarified that oxyhemoglobin is photodissociated in the ultrafast time scale within 50 fs. ${ }^{3,10-14}$ However, the dynamics after the photodissociation of heme protein has been controversial as described below.

Petrich et al. studied the photodissociation process of $\mathrm{HbO}_{2}$ using the pump-probe method, pumping at the visible absorption band (Q-band) and probing at the ultraviolet absorption band (Soret band). ${ }^{10}$ The photolyzed hemoglobin generated in the ultrafast photodissociation was thought to consist of two intermediates of $\mathrm{Hb}^{*}$ and $\mathrm{Hb}^{*}{ }_{\mathrm{II}}$. $\mathrm{Hb}^{*}{ }_{\mathrm{I}}$ has a partially domed structure working as a barrier for recombination of the oxygen molecule, and transforms into the other intermediate of deoxyhemoglobin in $300 \mathrm{fs}$. $\mathrm{Hb}^{*}$ II has a planar structure, being easily recombined with photodissociated oxygen in $3.2 \mathrm{ps}$. In their later study, the ultrafast dynamics of photoexcited $\mathrm{HbCO}$, $\mathrm{HbNO}$, and deoxyHb was studied by using the resonance Raman method. ${ }^{15}$ The result shows that the photoexcited state ${ }^{1} \mathrm{Q}$ decays sequentially: ${ }^{1} \mathrm{Q} \rightarrow \mathrm{Hb}^{*}{ }_{\mathrm{I}} \rightarrow \mathrm{Hb}^{*}{ }_{\mathrm{II}} \rightarrow \mathrm{Hb}$ ground state. The lifetimes of these states were determined as $<50 \mathrm{fs}, \approx 300 \mathrm{fs}$, and $\approx 3$ ps for ${ }^{1} \mathrm{Q}, \mathrm{Hb}^{*}$, and $\mathrm{Hb}^{*}{ }_{\mathrm{II}}$, respectively. $\mathrm{Hb}^{*}{ }_{\mathrm{I}}$ state results from an ultrafast iron-to-porphyrin ring charge-transfer process, and $\mathrm{Hb}_{\mathrm{II}}$ state arises from porphyrin ring-to-iron back charge transfer (CT) to produce a porphyrin ground-state configuration with a nonequilibrium iron d-orbital population.

* To whom correspondence should be addressed. Tel.: 88635712121 , ext 56197. Fax: 8863 5725230. E-mail: yabushita@mail.nctu.edu.tw.

National Chiao-Tung University.

*apan Science and Technology Agency.

${ }^{\S}$ University of Electro-Communications.

"I Osaka University.
The CT character of $\mathrm{Hb}_{\mathrm{I}}$ explains the decay caused by ultrafast intersystem crossing of this state, providing a key mechanism for ultrafast photolysis. ${ }^{16,17}$

Molecular dynamics simulation on Mb by Henry et al. showed that the decay of vibrational temperature occurs on a few picoseconds time scale and several tens of picoseconds. ${ }^{25}$ Mizutani et al. observed thermal relaxation of photolyzed heme as 3 and 25 ps using resonant Raman measurement of MbCO. ${ }^{18}$ Pump-probe spectroscopy of $\mathrm{Mb}$ was performed by Kholodenko et al. pumping at Soret band and probing at Q-band. ${ }^{19}$ It showed that the photoexcited $\mathrm{Mb}$ decays to the ground electronic state in two ultrafast electronic relaxation steps, one shorter than $100 \mathrm{fs}$ and the other of a few hundred femtoseconds. Further relaxations of a few picoseconds and 15 ps components were assigned to the vibrational cooling in the ground electronic state. Subsequently, very detailed studies have been performed to explain the ultrafast dynamics obtained by Champion's group for myoglobin and its ligated species. ${ }^{19,20}$ They performed timeresolved measurements probing at Soret band and pumping at Soret band or Q-band. It was concluded that hot ligand-bound heme system contributes the transient optical response with a $\sim 1$ ps time constant, as the six-coordinate heme system cools. The six-coordinate metMb sample was showing very similar $(\sim 1 \mathrm{ps})$ behavior with no detectable photodissociation. On the other hand, the five-coordinate deoxyheme displayed a biphasic signal with time constants of $\sim 0.5$ and $\sim 2.4$ ps.

In the present study, an ultrafast time-resolved pump-probe signal of $\mathrm{HbO}_{2}$ was observed pumping at the Soret band and probing at Q-band with an ultrashort visible laser pulse in a broad visible spectral range. The photodissociation of $\mathrm{HbO}_{2}$ known to be faster than $50 \mathrm{fs}$ was time-resolved as $45 \pm 5 \mathrm{fs}$ in this work using the ultrashort pulse. Simultaneous measurements at as many as 128 probe wavelengths were performed using a multichannel detector array to obtain ultrafast nearly continuous difference spectrum after the photoexcitation of 
oxyhemoglobin. Thus, we obtained for the first time the spectra of ultrashort lifetime electronic excited state of $\mathrm{HbO}_{2}$ in the visible range extending from $523 \mathrm{~nm}\left(19109 \mathrm{~cm}^{-1}\right)$ to $719 \mathrm{~nm}$ (13 $914 \mathrm{~cm}^{-1}$ ). Observed $\sim 1$ ps time scale decays can be explained following previous works shown above.

\section{Experimental Methods}

Materials. A phosphate buffer stabilized at $(\mathrm{pH}=7.7,0.1$ M) was used to dissolve excess amount of hemoglobin from bovine blood (Sigma-Aldrich). A filter (Millex; Millipore) was inserted over the head of an injection syringe to remove undissolved residue of hemoglobin passing through the filter. Sodium thiosulfate (Tokyo Chemical Industry, Ltd.) was added to the solution to have molecular density of $0.1 \mathrm{M}$ in the sample solution to reduce $\mathrm{Hb}$. The density of the solution was carefully adjusted while monitoring their absorption spectra using an ultraviolet-visible-near-infrared (UV-vis-NIR) scanning spectrophotometer (UV-3101PC; Shimadzu) and the optical density at $540 \mathrm{~nm}$ was adjusted to be about 1.0 in a $1 \mathrm{~mm}$ glass cell (6210-27501; GL Science) as shown in Figure 1.

Ultra-Short-Pulse Laser System. As the light source in this work, we used a Ti:sapphire laser system (FemtoSource (sPRO); FemtoLasers) with an eight-path bow-tie amplifier (Femtopower; FemtoLasers). The amplifier generates pulses with a width of $20 \mathrm{fs}$, pulse energy of $30 \mu \mathrm{J}$, a spectral range of $750-870 \mathrm{~nm}$, and a repetition rate of $1 \mathrm{kHz}$. A type-I $\beta$-barium borate (BBO) crystal was used to produce second harmonic generation (SHG) of the fundamental pulse. The generated SHG pulse of $395 \mathrm{~nm}$ was compressed using a prism pair to be used as the pump pulse in the pump-probe measurements. A portion of the fundamental pulse remains in the light path of the pump beam after generating the SHG pulse. One prism of the prism pair for SHG pulse compression displaces this residual fundamental pulse. After passing through another prism, the fundamental pulse was compressed to $20 \mathrm{fs}$. Focusing the compressed fundamental pulse on a sapphire plate, the spectral range of the fundamental pulse was broadened to have a visible broadband spectrum via self-phase modulation in the sapphire plate. This visible broadband pulse was the probe pulse of the pump-probe measurements in this work. The chirp of the visible probe pulse caused by the material dispersion was suppressed using reflective optics in the probe light path from white light generation to the sample cell. The time resolution of the pump-probe measurement was estimated to be $10 \mathrm{fs}$ from the duration of coherent artifacts that appear at zero delay (see Figure $2 b$ ).

An electric stepper motor scanned the time delay of the probe pulse with respect to the pump pulses with a 1 fs step. The pump-probe signal detection system consisted of a polychromator (300 grooves/mm, $500 \mathrm{~nm}$ blazed) coupled to avalanche photo detectors via a 128-channel fiber bundle. Signal-to-noise ratio of the signal was improved by lock-in detection using a multichannel lock-in amplifier, which detects signals simultaneously over the entire spectrum. ${ }^{22}$ All measurements were performed at room temperature $(294 \pm 1 \mathrm{~K})$.

\section{Results and Discussion}

Stationary Spectrum of Sample and Laser Spectrum. Figure 1 shows the laser spectrum and the absorption spectrum of the oxyhemoglobin sample dissolved in water with a phosphate buffer stabilized at $\mathrm{pH}$ 7.7. The absorption spectrum of $\mathrm{HbO}_{2}$ has two peaks at $541 \mathrm{~nm}\left(18484 \mathrm{~cm}^{-1}\right)$ and $576 \mathrm{~nm}$ $\left(17360 \mathrm{~cm}^{-1}\right)$. The peak at $635 \mathrm{~nm}\left(15750 \mathrm{~cm}^{-1}\right)$ is the absorption peak of $\mathrm{Hb}^{3+}$ (metHb). The peak locations are in good agreement with those in the literature..$^{23,24}$ The probe pulse

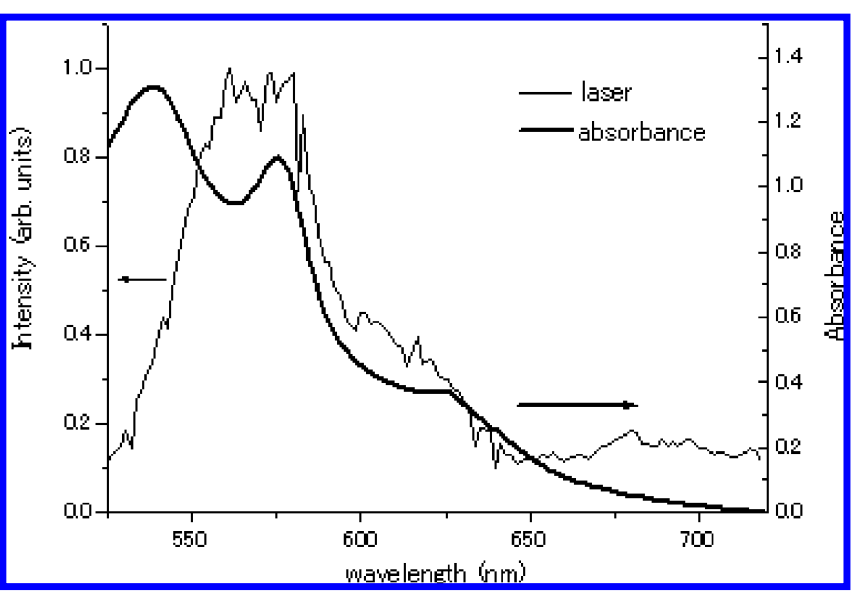

Figure 1. Laser spectrum (thin line) and stationary absorption spectrum of oxyhemoglobin solution sample (thick line).

spectrum extends from 520 to $720 \mathrm{~nm}$ with a peak around 570 $\mathrm{nm}$ that overlaps well with the stationary absorption spectrum of the $\mathrm{HbO}_{2}$ sample.

Overview of the Time-Dependent Difference Absorbance and Time-Resolved Difference Absorption Spectrum over the Full Time Range of Observations from -70 fs to 2 ps. Several real-time traces of difference absorbance at 554, 586, 619,651 , and $683 \mathrm{~nm}$ in the probe delay ranges from -70 to $1930 \mathrm{fs}$ and from -70 to $330 \mathrm{fs}$ are shown in Figure 2, a and $\mathrm{b}$, respectively.

In the real-time trace data, we found that the intensities of some finite signals decrease with the delay toward negative direction at probe wavelengths (frequencies) in the probe spectral range from $512 \mathrm{~nm}\left(19531 \mathrm{~cm}^{-1}\right)$ to $606 \mathrm{~nm}\left(16502 \mathrm{~cm}^{-1}\right)$. These signals disappear almost completely at a delay of -70 fs. This is an unexpected finding from the viewpoint of causality. The signal of the pump-probe experiment is induced by the third-order nonlinearity generated by the intense pump field and the weak probe field interacting with the sample. The unexpected signal is caused by so-called perturbed free induction decay process. It is due to the intense second field of the pump pulse diffracted by the grating produced by the interference between the macroscopic polarization generated by the preceding probe pulse and the pump (first) field. Therefore, the perturbed free induction decay is due to the third-order polarization caused by the nonlinearity in the temporal ordering of probepump-pump. The signal appears as long as the coherent macroscopic polarization induced by the probe field lasts for the electronic dephasing time.

Near the zero delay time there are very intense spiky signals whose profiles periodically change from dispersive type profiles to absorptive or emissive type profiles and vice versa with an increase (decrease) in the probe photon energy (wavelength). This is due to the coherent artifacts that arise when the temporal ordering of the interacting fields is pump-probe-pump. Therefore, this appears only when the pump and probe pulses overlap in time.

For the positive delays, all of the traces in Figure 2a have oscillatory features superimposed on the slowly varying difference absorbance $\Delta A(\lambda, t)$ at the probe wavelength $\lambda$ as a function of the pump probe delay $t$. This is because when the pump pulse reaches the sample before the probe pulse, there are slow electronic decay dynamics together with oscillatory structures due to molecular vibrations. We analyzed the electronic decay dynamics in the positive delay region. Previous studies ${ }^{3,10-14}$ showed that the first reaction just after the photoexcitation of 


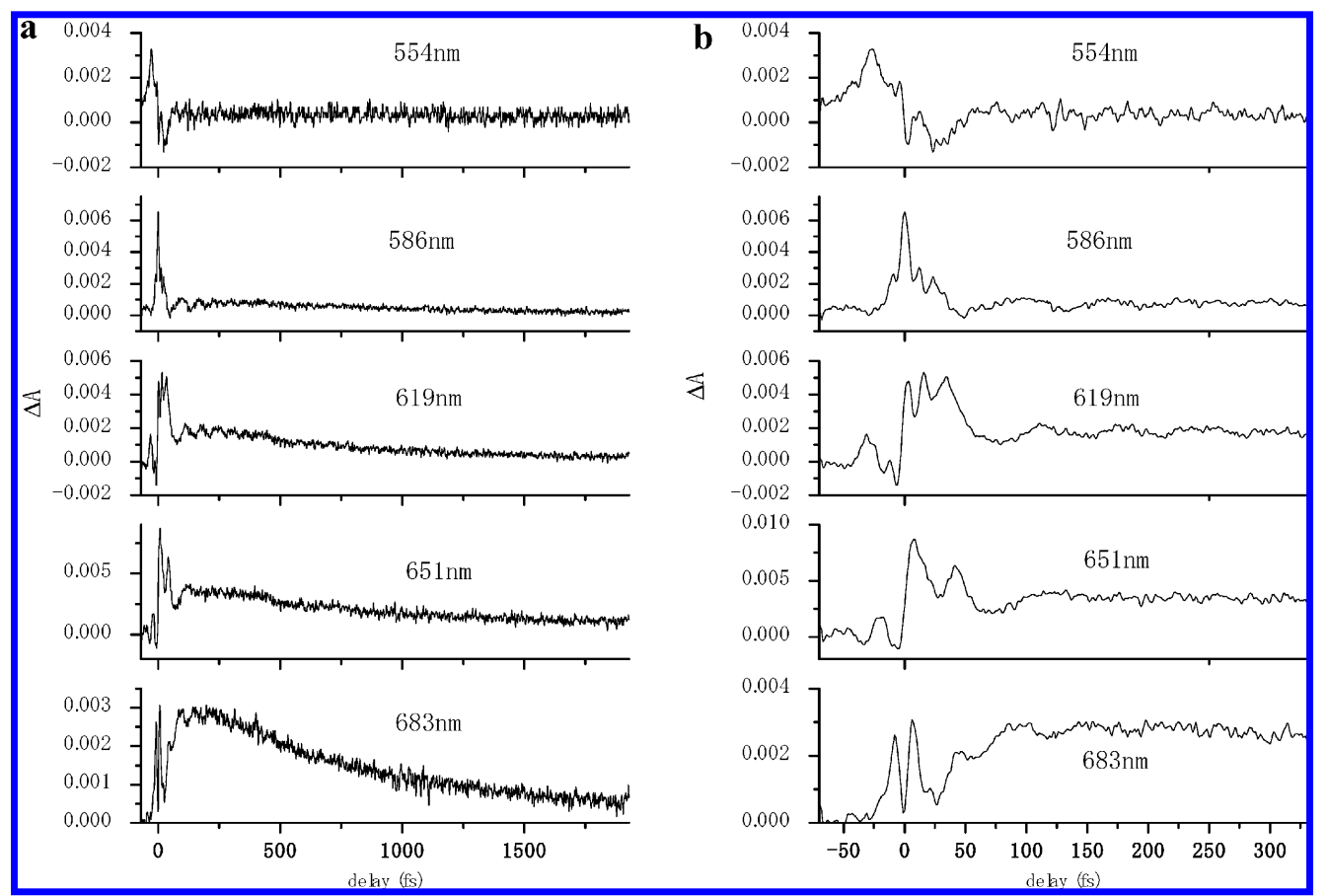

Figure 2. Several real-time traces of difference absorbance at $554,586,619,651$, and $683 \mathrm{~nm}$ in probe delay ranges (a) from -70 to $1930 \mathrm{fs}$ and (b) from -70 to $330 \mathrm{fs}$.

$\mathrm{HbO}_{2}$ is photodissociation and finishes faster than $50 \mathrm{fs}$. In the current measurement region up to a few picoseconds, the photolyzed heme protein was reported to show several hundred femtosecond and a few picosecond decays.

To determine the decay time constants and the spectra of the species, we analyzed the data in the following way. First, the $\triangle A(\lambda, t)$ traces of 10 neighboring channels were averaged. Then, the averaged traces of $\Delta A(\lambda, t)$ were smoothed using a moving gate. After smoothing the signal, the decay can be expressed by the following equation that contains three decaying components:

$$
\begin{aligned}
& \Delta A(\lambda, t)=\Delta A_{1}(\lambda) \mathrm{e}^{-t / \tau_{1}}+\Delta A_{2}(\lambda) \mathrm{e}^{-t / \tau_{2}}+ \\
& \Delta A_{3}(\lambda) \mathrm{e}^{-t / \tau_{3}} \quad\left(\tau_{1}<\tau_{2}<\tau_{3}\right)
\end{aligned}
$$

Fine structures on time-resolved spectra were distinct earlier than 30 fs delay because of coherent artifact. Therefore, we performed exponential fit in the time range 60-1930 fs avoiding the effect of the coherent artifact. Fitting the time trace by the above equation in the time range $60-1930 \mathrm{fs}$, the time constants were determined to be $\tau_{1}=45 \pm 5 \mathrm{fs}, \tau_{2}=676 \pm 75 \mathrm{fs}$, and $\tau_{3}>2$ ps. The spectra of $\Delta A_{1}(\lambda), \Delta A_{2}(\lambda)$, and $\Delta A_{3}(\lambda)$ were obtained by fitting the simulated curves with the observed spectra as shown in Figure 3a.

The shortest time constant $\tau_{1}=45 \pm 5 \mathrm{fs}$ is attributed to the transition from photoexcited $\mathrm{HbO}_{2}$ to the photolyzed $\mathrm{Hb}$. As discussed in the previous work, ${ }^{21}$ metHb cannot bind oxygen and does not have any ultrafast dynamics faster than $\sim 1 \mathrm{ps}$. Both photodissociation and heme-iron out-of-plane movement occur very rapidly ( $<50 \mathrm{fs}$ ) to create the unligated species. Several research groups have studied the ultrafast dynamics, ${ }^{10,15,16,18,20,21}$ but the lifetime of the excited state could not be resolved precisely-only its upper limit has been determined to be $50 \mathrm{fs}$. This has been measured for the first time in the present study. The $300 \mathrm{fs}$ decay reported in previous works was not observed in this work, which

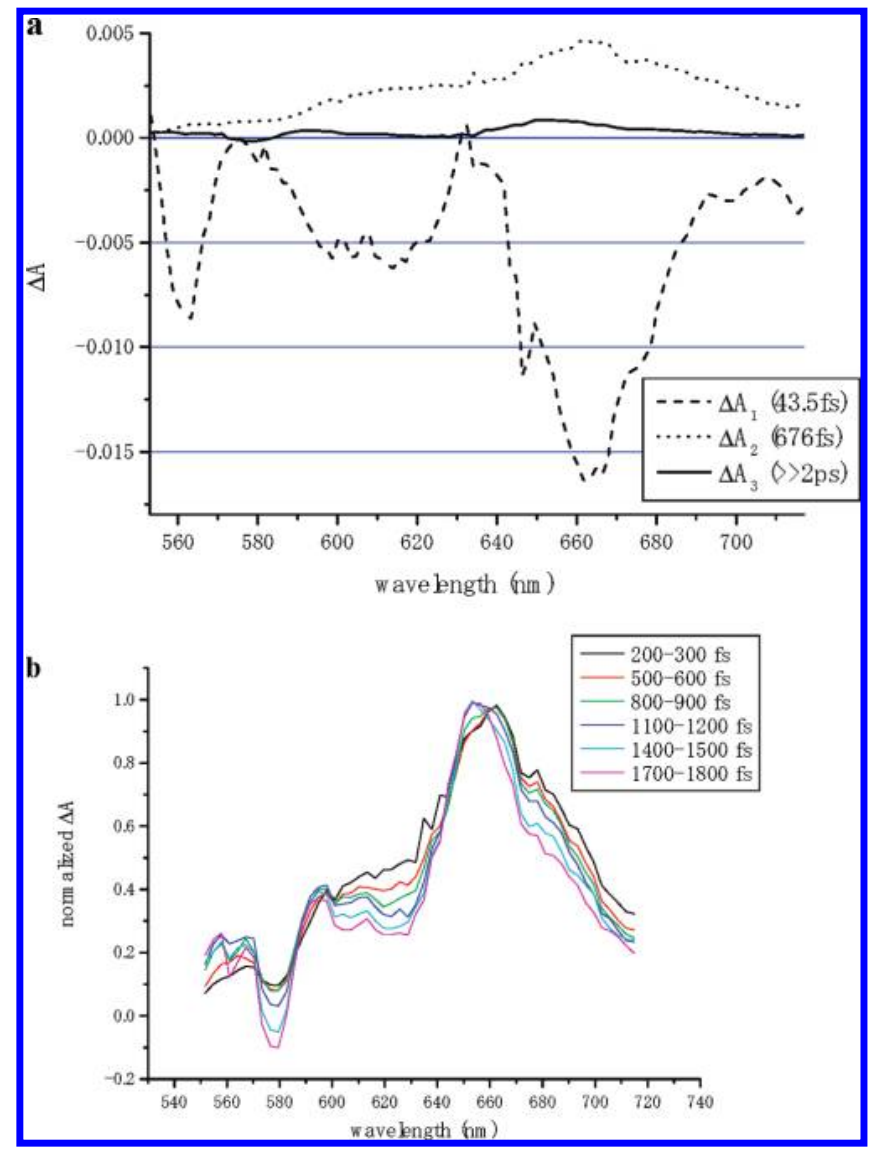

Figure 3. (a) Spectra of $\Delta A_{1}(\lambda), \Delta A_{2}(\lambda)$, and $\Delta A_{3}(\lambda)$ obtained by fitting analysis. (b) Normalized difference absorption spectra $\Delta A(\lambda)$ averaged for $100 \mathrm{fs}$.

is thought to be because the sample solution in this work has high density compared with those previous works.

The second shortest time constant $\tau_{2}=680 \pm 80 \mathrm{fs}$ corresponds to the faster time constant of $\sim 0.5$ ps observed for five-coordinate deoxyMb in the Soret band. ${ }^{21}$ This time constant 


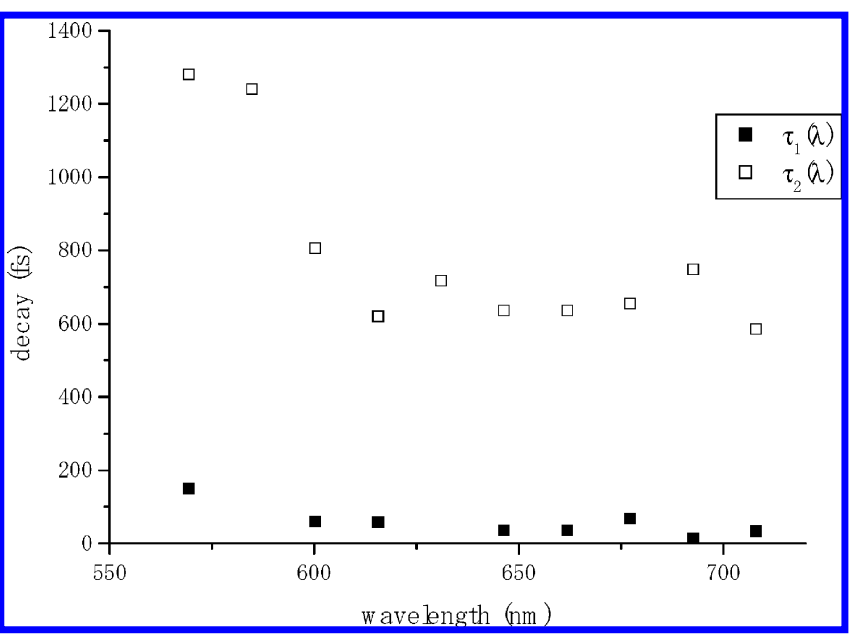

Figure 4. Probe wavelength dependences of $\tau_{1}(\lambda)(\square)$ and $\tau_{2}(\lambda)(\square)$.

is corresponding to the process of first vibrational cooling of the hot heme group in deoxyHb. A time constant corresponding to that with a few picoseconds due to the second vibrational cooling process observed in previous works ${ }^{18-21,25}$ was not accurately determined in this experiment due to the limited time window of $1.9 \mathrm{ps}$. The third shortest time constant $\tau_{3}>2 \mathrm{ps}$ includes the picosecond vibrational cooling process. Timeresolved difference absorption spectra $\Delta A(\lambda)$ was averaged for $100 \mathrm{fs}$ and normalized at peak around $660 \mathrm{~nm}$. The result is plotted in Figure 3b. It shows narrowing and blue shift, implying the vibrational cooling in the subpicosecond and a few picoseconds time region.

The lifetimes, $\tau_{1}\left(\lambda_{\text {probe }}\right)$ and $\tau_{2}\left(\lambda_{\text {probe }}\right)$, are dependent on the probe wavelength $\lambda_{\text {probe }}$ (see Figure 4 ). This is partly due to the limited signal-to-noise ratio and partly due to the mixing of the coherent spike in this time range. However, the values of $\tau_{1}\left(\lambda_{\text {probe }}\right)$ in the probe wavelength range between 600 and 710 $\mathrm{nm}$ are close to each other, having an average value of $45 \pm 5$ fs. They have much longer lifetimes when $\lambda_{\text {probe }}<600 \mathrm{~nm}$. The same trends were also found for $\tau_{2}\left(\lambda_{\text {probe }}\right) . \tau_{2}(\omega)$ has an average value of $680 \mathrm{fs}$ when $\lambda_{\text {probe }}>600 \mathrm{~nm}$, whereas the lifetimes are much longer at shorter wavelength ranges. This may be due to the cross talk between the signals of vibrational cooling process of deoxyHb $(\sim 0.5 \mathrm{ps})$ and metHb $(\sim 1 \mathrm{ps})$. The contribution of the cooling process of metHb is more around the absorption peak of metHb of $570 \mathrm{~nm}$.

Time-Resolved Spectrum and Time-Dependent Difference Absorbance in the Short Probe Time Range between 0 and 100 fs. From the difference spectra $\Delta A_{1}(\lambda)$ and $\Delta A_{2}(\lambda)$ of the components with respective lifetimes of $\tau_{1}$ and $\tau_{2}$ shown in Figure 3 , it can be concluded that the major contribution is due to $\Delta A_{2}(\lambda)$ throughout the entire probe spectral range. The dynamics at probe wavelength $\lambda_{\text {probe }}<600 \mathrm{~nm}$ seems to differ from those in the other range. Therefore, we analyze real-time spectral changes in broadband in the early delay region of $0-100 \mathrm{fs}$ where the process is largely affected by ultrafast photodissociation that finishes within 50 fs.

We studied the spectral changes that occur during the ultrafast photodissociation process. Figure 5 shows the difference spectra $\Delta A(\lambda)$ in the delay region of $0-100 \mathrm{fs}$ averaged over $10 \mathrm{fs}$ to observe ultrafast spectral changes. The observed positive difference absorption spectrum $\Delta A(\lambda)$ is considered to be due to induced absorption from photoexcited $\mathrm{HbO}_{2}$. The spectra show oscillations reflecting wavepacket motion on the potential energy surface of the photoexcited $\mathrm{HbO}_{2}$ during the ultrafast

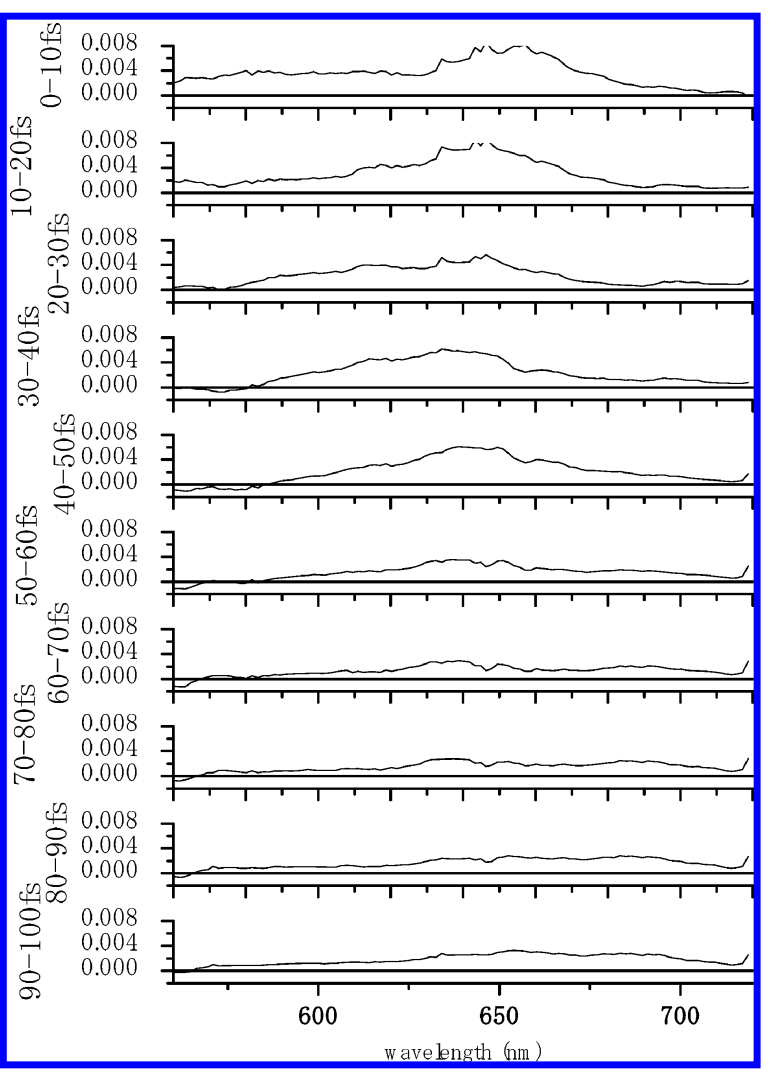

Figure 5. Difference absorption spectra $\Delta A(\lambda)$ averaged for $10 \mathrm{fs}$.

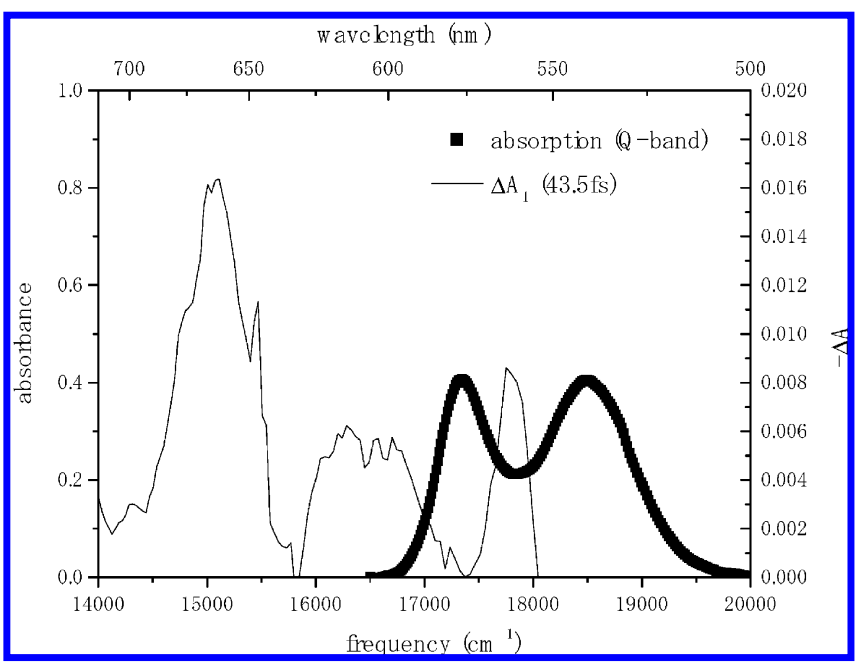

Figure 6. Stationary absorption spectrum of the Q-band of oxyhemoglobin (filled squares) and $\Delta A_{1}(\lambda)$ (solid curve) shown in Figure 3.

photodissociation process, which is finished in $70 \mathrm{fs}$ and allows the oscillation of $\mathrm{O}=\mathrm{O}$ stretching molecular vibration affect the absorption spectrum of porphyrin only 1.2 times.

Figure 6 shows the absorption spectrum of the Q-band of $\mathrm{HbO}_{2}$ after subtracting the background from the measured spectrum (Figure 1). The negative $\Delta A_{1}(\omega)$ spectrum has two peaks at $665 \mathrm{~nm}\left(15070 \pm 7 \mathrm{~cm}^{-1}\right)$ and $615 \mathrm{~nm}(16263 \pm 23$ $\mathrm{cm}^{-1}$ ) separated by $1197 \pm 30 \mathrm{~cm}^{-1}$, which is a mirror image of the Q-band absorption spectrum of $\mathrm{HbO}_{2}$ with peaks at 541 $\mathrm{nm}\left(18470 \pm 3 \mathrm{~cm}^{-1}, Q_{\mathrm{v}}\right.$ band $)$ and $576 \mathrm{~nm}\left(17360 \pm 3 \mathrm{~cm}^{-1}\right.$, $\mathrm{Q}_{0}$ band) separated by $1111 \mathrm{~cm}^{-1}$.

Induced absorption of an excited state has positive value in absorption change. Negative absorption change can originate from "transient bleaching due to ground-state depletion" or "stimulated emission associated with the transition from the 
excited state to the ground state". $\Delta A_{1}$ has negative value and does not overlap with Q-band of the ground state; therefore, it was assigned to the stimulated emission from Q-band.

$\mathrm{Q}_{\mathrm{v}}$ band and $\mathrm{Q}_{0}$ band have similar amplitude in the groundstate absorption spectrum. However, a peak of $\Delta A_{1}$ at $665 \mathrm{~nm}$, which corresponds to stimulated emission from $\mathrm{Q}_{0}$ state, is higher than another at $615 \mathrm{~nm}$ (stimulated emission from $\mathrm{Q}_{\mathrm{v}}$ band). One of the possible explanations for this phenomenon is that the transition probability from Soret band to $\mathrm{Q}_{0}$ band is higher than that to $\mathrm{Q}_{\mathrm{v}}$ band. Another possibility is because of ultrafast transition from $\mathrm{Q}_{\mathrm{v}}$ band to $\mathrm{Q}_{0}$ band which proceeds much faster than 45 fs.

As for the other peak of $\Delta A_{1}(\omega)$ at $562 \mathrm{~nm}(17800 \pm 3$ $\mathrm{cm}^{-1}$ ), this peak was thought to be reflecting the appearance of unligated hemoglobin absorption band after ultrafast photodissociation because this peak frequency agrees with the absorption band of deoxyhemoglobin..$^{23,24}$

$\Delta A_{2}$ and $\Delta A_{3}$ have positive values reflecting induced absorption of hot ground states whose lifetimes are $\tau_{2}$ and $\tau_{3}$, respectively. Therefore, the spectra of $\Delta A_{2}$ and $\Delta A_{3}$ seem to be different from the stationary absorption spectrum of (thermally cooled) deoxyHb.

\section{Conclusions}

In this study, ultrafast time-resolved pump-probe spectroscopy in the visible spectral range was performed using an ultrashort visible laser pulse. The broadband visible laser pulse enabled to observe the pump-probe signal in the spectral range of 523-719 nm. The pump-probe signal was simultaneously detected with high sensitivity at 128 probe frequencies using a multichannel detector array. The simultaneous measurement at multiple probe wavelengths could provide the ultrafast spectral changes after the photoexcitation of oxyhemoglobin in a short measurement time. This helps minimize the effects of laser damage to the sample and laser intensity instability.

The time constant of the primary process (the upper limit of which had been previously estimated) was determined for the first time as $45 \pm 5$ fs. Longer time constant of $680 \pm 80 \mathrm{fs}$ obtained in this work was attributed to the decay time of photolyzed $\mathrm{Hb}$ because it is the same as the one reported in previous works. The spectrum of the ultrashort lifetime $(<50$ fs) photoexcited $\mathrm{HbO}_{2}$ was determined in the spectral range 523 $\mathrm{nm}\left(19100 \mathrm{~cm}^{-1}\right)$ to $719 \mathrm{~nm}\left(13910 \mathrm{~cm}^{-1}\right)$ in the visible range. Time-resolved difference absorption spectra were studied in the early delay region to study the spectral change affected by ultrafast photodissociation process that finished within 50 fs. Observed real-time spectral change showed oscillatory motion reflecting wavepacket motion in the potential energy surface of the photoexcited $\mathrm{HbO}_{2}$ during the ultrafast photodissociation.
Acknowledgment. This work was supported by ICORP program of Japan Science and Technology Agency (JST), National Science Council of the Republic of China, Taiwan (NSC 98-2112-M-009-001-MY3), and the grant MOE ATU Program in NCTU. This work was also supported in part by a Grant-in-Aid for Scientific Research from the Japan Society for the Promotion of Science (JSPS-GASR-14002003).

\section{References and Notes}

(1) Chernoff, D. A.; Hochstrasser, R. M.; Steele, A. W. Proc. Natl. Acad. Sci. U.S.A. 1980, 77, 5606.

(2) Mizutani, Y.; Kitagawa, T. J. Phvs. Chem. B 2001, 105, 10992.

(3) Martin, J. L.; Migus, A.; Poyart, C.; Lecarpentier, Y.; Astier, R.; Antonetti, A. Proc. Natl. Acad. Sci. U.S.A. 1983, 80, 173.

(4) Martin, J. L.; Migus, A.; Poyart, C.; Lecarpentier, Y.; Astier, R.; Antonetti, A. EMBO J. 1983, 2, 1815.

(5) Martin, J. L.; Migus, A.; Poyart, C.; Lecarpentier, Y.; Antonetti, A. Hemoglobin; Brussels University Press: Brussels, 1984.

(6) Martin, J. L.; Migus, A.; Poyart, C.; Lecarpentier, Y.; Astier, R.; Antonetti, A. Ultrafast Phenomena IV; Springer-Verlag: Berlin, 1984.

(7) Cornelius, P. A.; Hochstrasser, R. M.; Steele, W. A. J. Mol. Biol.

1983, 163, 119-128.

(8) Friedman, J. M.; Scott, T. W.; Fisanick, G. J.; Simon, S. R.; Findsen,

E. W.; Ondrias, M. R.; Macdonald, V. W. Science 1985, 229, 187.

(9) Houde, D.; Petrich, J. W.; Rojas, O. L.; Poyart, C.; Antonetti, A.; Martin, J. L. Ultrafast Phenomena V; Springer-Verlag: Berlin, 1986.

(10) Petrich, J. W.; Poyart, C.; Martin, J. L. Biochemistry 1988, 27, 4049.

(11) Baldwin, J. M.; Chothia, C. J. Mol. Biol. 1979, 129, 175.

(12) Franzen, S.; Bohn, B.; Poyart, C.; DePillis, G.; Boxer, S. G.; Martin, J. L. J. Biol. Chem. 1995, 270, 1718.

(13) Franzen, S.; Bohn, B.; Poyart, C.; Martin, J. L. Biochemistry 1995, $34,1224$.

(14) Perutz, M. F. Annu. Rev. Biochem. 1979, 48, 327.

(15) Franzen, S.; Kiger, L.; Poyart, C.; Martin, J. L. Biophys. J. 2001, 80,2372

(16) Greene, B. I.; Hochstrasser, R. M.; Weisman, R. B.; Eaton, W. A. Proc. Natl. Acad. Sci. U.S.A. 1978, 75, 5255.

(17) Waleh, A.; Loew, G. H. J. Am. Chem. Soc. 1982, 104, 2346.

(18) Mizutani, Y.; Kitagawa, T. Science 1997, 278, 443.

(19) Kholodenko, Y.; Volk, M.; Gooding, E.; Hochstrasser, R. M. Chem. Phys. 2000, 259, 71.

(20) Ye, X.; Demidov, A.; Rosca, F.; Wang, W.; Kumar, A.; Ionascu, D.; Zhu, L.; Barrick, D.; Wharton, D.; Champion, P. M. J. Phys. Chem. 2003, 107, 8156

(21) Ye, X.; Demidov, A.; Champion, P. M. J. Am. Chem. Soc. 2002, $124,5914$.

(22) Ishii, N.; Tokunaga, E.; Adachi, S.; Kimura, T.; Matsuda, H.; Kobayashi, T. Phys. Rev. A 2004, 70, 023811.

(23) Anderson, R. R.; Parrish, A. J. Invest. Dermatol. 1981, 77, 13.

(24) Asimov, M. M.; Asimov, R. M.; Rubinov, A. N. J. Appl. Spectrosc. 1998, 65, 919 .

(25) Henry, E. R.; Eaton, W. A.; Hochstrasser, R. M. Proc. Natl. Acad. Sci. U.S.A. 1986, 83, 8982.

(26) Eaton, W. A.; Hanson, L. K.; Stephens, P. J.; Sutherland, J. C.; Dunn, J. B. R. J. Am. Chem. Soc. 1978, 100, 4991.

JP103593Q 